\title{
MOSCAS-DAS-FRUTAS (DIPTERA, TEPHRITIDAE) EM MUNICÍPIOS COM SISTEMA DE MITIGAÇÃO DE RISCO PARA ANASTREPHA GRANDIS MACQUART
}

\section{L.R.S. Rabelo, V.R.S. Veloso, A.D.F. Rios, C.S. Queiroz, F.H.S. Meshima}

Universidade Federal de Goiás, Escola de Agronomia e Engenharia de Alimentos, Departamento de Fitossanidade, CP 131, CEP 74690-900, Goiânia, GO, Brasil. E-mail: Lilian.agrodefesa@gmail.com.br

\author{
RESUMO
}

\begin{abstract}
O objetivo do estudo foi verificar a presença de Anastrepha grandis e identificar demais espécies de moscas-das-frutas coletadas em armadilhas modelo MacPhail instaladas em áreas de cucurbitáceas sob Sistema de Mitigação de Risco (SMR) para A. grandis na região Vale do São Patrício, GO, de novembro de 2004 a outubro de 2009. Um total de 812 espécimes de Anastrepha foram capturadas, sendo 639 machos (78,7\%) e 173 fêmeas (21,3\%). Destas foram identificadas 14 espécies de Anastrepha, ocorrentes em todas as estações do ano. A. dissimilis Stone, A. quiinae Lima e A. pickeli Lima foram registradas pela primeira vez em Goiás e primeiro registro de $A$ grandis, com baixa prevalência, nos municípios de Jaraguá e Uruana, constituintes da área de SMR, indicando ótimas condições para manutenção de área de baixa prevalência. A. manihoti Lima (34,10\%) foi a espécie mais frequente na região, seguida de A. obliqua (Macquart) (19,65\%) e A. pickeli (13,87\%).
\end{abstract}

PALAVRAS-CHAVE: Insecta, Anastrepha, flutuação populacional, baixa prevalencia.

\section{ABSTRACT}

FRUIT FLIES (DIPTERA, TEPHRITIDAE) IN MUNICIPALITIES WITH A RISK MITIGATION SYSTEM FOR ANASTREPHA GRANDIS MACQUART. The objective of this study was verify the presence of $A$. grandis and identify other fruit-fly species collected in MacPhail traps, installed in areas of Cucurbitaceae under a risk mitigation system for Anastrepha grandis Macquart in the Vale do São Patrício Region, state of Goiás, Brazil, from November 2004 to October 2009. A total of 812 specimens of Anastrepha were captured, of which 639 were males (78.7\%) and 173 were females (21.3\%). Fourteen species of Anastrepha were identified occurring in all seasons of the year. A. dissimilis Stone, A. quiinae Lima and A. pickeli Lima were recorded for the first time in the state of Goiás, and $A$. grandis and was recorded for the first time, with a low prevalence, in the municipalities of Jaraguá and Uruana, which belong to the risk mitigation system area, indicating good conditions for maintaining an area of low prevalence status. A. manihoti Lima (34.10\%) was the most frequent species in the region, followed by A. obliqua (Macquart) $(19.65 \%)$ and A. pickeli Lima (13.87\%).

KEY WORDS: Insecta, Anastrepha, population fluctuation, low prevalence area.

A região do Vale do São Patrício, localizada na mesorregião central do Estado de Goiás, tem se destacado na exploração de olerícolas. Situados nesta região, os municípios de Carmo do Rio Verde, Jaraguá, Itapuranga e Uruana têm como principais atividades econômicas os cultivos de abóbora (Cucurbita maxima Duchesne), melão (Cucumis melo L.) e melancia (Citrullus lanatus Thunb. Masnsf.), voltados para exportação. Em 2010, um total de 213.600 toneladas foi produzido nesta região, das quais $3.766,27$ toneladas foram exportadas para Argentina (Goiás, 2010; Goiás, 2013).

As moscas-das-frutas (Diptera: Tephritidae) são consideradas como uma das mais importantes pragas na fruticultura mundial. No Brasil, os gêneros Ceratitis e Anastrepha são os de maior importância econômica, onde o primeiro é representado por apenas uma espécie, Ceratitis capitata, e o segundo por 114 espécies, de acordo com Zucchi (2008). Em Goiás, 19 espécies de Anastrepha estão catalogadas (Veloso et al., 2000; Zucchi, 2007; 2008). Na Região do Vale do São Patrício, as espécies $A$. fraterculus Wiedemann e $A$. zenildae Zucchi foram registradas pela primeira vez por FELIPE (2004).

A mosca-das-cucurbitáceas-sul-americana A.grandis é especialista em plantas da família Cucurbitaceae, com distribuição ampla na América do Sul e Central, no Panamá (MALAvasi et al., 2000; NorRbom et al., 2012). É considerada como praga quarentenária, pelo Departamento de Agricultura dos Estados Unidos, Argentina e Uruguai para frutos de cucurbitáceas provenientes de países onde há 
a presença dessa mosca (NorRBOm et al., 2012). No Brasil, a ocorrência de $A$. grandis é relatada para os estados do Rio Grande do Sul, Santa Catarina, Paraná, São Paulo, Rio de Janeiro, Espírito Santo, Mato Grosso, Mato Grosso do Sul e Goiás (ZucchI, 1988; Norrbom, 1991; Zucchi, 2000b; Veloso et al., 2005; ZucchI, 2007; 2008) e na Bahia apenas no extremo norte (BONDAR, 1950).

Com o objetivo de atender às exigências fitossanitárias específicas de países importadores de frutos frescos de cucurbitáceas que classificam $A$. grandis como uma praga quarentenária, no Brasil, são aplicadas medidas sob um enfoque de Sistemas de Mitigação de Risco (BRAsIL, 2006). O Estado de Goiás implantou e obteve o reconhecimento oficial, pelo Ministério da Agricultura, Pecuária e Abastecimento, doSMR para A. grandis, em cultivos decucurbitáceas, a partir de 2006, nos municípios de Carmo do Rio Verde, Itapuranga, Jaraguá e Uruana, na região do Vale do São Patrício. O sistema possibilitou a exportação para Argentina de frutos frescos de melancia, melão e abóbora, oriundos desses municípios.

Os objetivos deste trabalho foram verificar a presença de $A$. grandis e identificar demais espécies de moscas-das-frutas coletadas em armadilhas modelo MacPhail, em áreas de cucurbitáceas sob Sistema de Mitigação de Risco (SMR) para A. grandis.

Os levantamentos foram conduzidos na região do Vale do São Patrício, de novembro de 2004 a outubro de 2009, nos municípios de Carmo do Rio
Verde ( $15^{\circ} 21^{\prime} 14^{\prime \prime}$ S e $\left.49^{\circ} 41^{\prime} 16^{\prime \prime} \mathrm{W}\right), 629 \mathrm{~m}$ de altitude, Itapuranga $\left(15^{\circ} 33^{\prime} 43^{\prime \prime} \mathrm{S}\right.$ e $\left.49^{\circ} 56^{\prime} 56^{\prime \prime} \mathrm{W}\right), 651 \mathrm{~m}$ de altitude, Jaraguá $\left(15^{\circ} 45^{\prime} 25^{\prime \prime} \mathrm{S}\right.$ e $\left.49^{\circ} 20^{\prime} 02^{\prime \prime} \mathrm{W}\right), 666 \mathrm{~m}$

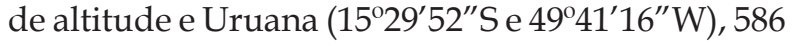
$m$ de altitude.

As moscas-das-frutas foram coletadas pelo programa SMR de A. grandis, implantado em Goiás para exportação de frutos de cucurbitáceas. Para implantação doSMR foi necessário o monitoramento ininterrupto por seis meses nos municípios propostos (BrASIL, 2006). Assim, de novembro de 2004 a junho de 2005, as armadilhas foram instaladas em pomares domésticos, próximos às áreas de cucurbitáceas. Posteriormente, para manutenção do sistema, a cada ano as armadilhas foram instaladas nas lavouras de cucurbitáceas, permanecendo durante os períodos de produção.

As armadilhas modelo McPhail, que continham como atrativo alimentar proteína hidrolisada de milho a $5 \%$, foram instaladas nas lavouras com densidade de uma armadilha para 5 hectares, fixadas sob um suporte de madeira a $50 \mathrm{~cm}$ do solo (BRASIL, 2006).

Em cada município, foram monitoradas propriedades que se cadastraram no SMR, junto à Agência Goiana de Defesa Agropecuária (AGRODEFESA) e à Superintendência Federal de Agricultura de Goiás (SFA/GO) (BRASIL, 2006). De 2004 a 2009, foram cadastradas 42 propriedades com área de aproximadamente1.120 hectares delavouras decucurbitáceas, totalizando 487 armadilhas.

Tabela 1 - Número de fêmeas e porcentual de espécies de Anastrepha coletadas em armadilhas modelo McPhail, na região do Vale do São Patrício (GO), de novembro de 2004 a outubro de 2009.

\begin{tabular}{|c|c|c|c|c|c|c|c|c|c|c|c|}
\hline \multirow{3}{*}{ Grupos infragenéricos } & \multirow{3}{*}{ Espécies } & \multicolumn{8}{|c|}{ Municípios } & \multirow{2}{*}{\multicolumn{2}{|c|}{ Total }} \\
\hline & & \multicolumn{2}{|c|}{$\begin{array}{l}\text { Carmo do } \\
\text { Rio Verde }\end{array}$} & \multicolumn{2}{|c|}{ Itapuranga } & \multicolumn{2}{|c|}{ Jaraguá } & \multicolumn{2}{|c|}{ Uruana } & & \\
\hline & & $\mathrm{N}^{\mathrm{o}}$ & $\%$ & $\mathrm{~N}^{\mathrm{o}}$ & $\%$ & $\mathrm{~N}^{\mathrm{o}}$ & $\%$ & $\mathrm{~N}^{\mathrm{o}}$ & $\%$ & $\mathrm{~N}^{\mathrm{o}}$ & $(\%)$ \\
\hline \multirow[t]{8}{*}{ fraterculus } & A. amita & 0 & 0,00 & 0 & 0,00 & 0 & 0,00 & 1 & 1,56 & 1 & 0,59 \\
\hline & A. distincta & 0 & 0,00 & 0 & 0,00 & 0 & 0,00 & 1 & 1,56 & 1 & 0,59 \\
\hline & A. fraterculus & 7 & 15,56 & 3 & 5,77 & 2 & 16,67 & 0 & 0,00 & 12 & 6,95 \\
\hline & A. obliqua & 8 & 17,78 & 10 & 19,23 & 4 & 33,33 & 12 & 18,75 & 34 & 19,65 \\
\hline & A. quiinae & 0 & 0,00 & 1 & 1,92 & 0 & 0,00 & 2 & 3,13 & 3 & 1,73 \\
\hline & A. sororcula & 0 & 0,00 & 0 & 0,00 & 0 & 0,00 & 1 & 1,56 & 1 & 0,59 \\
\hline & A. turpiniae & 0 & 0,00 & 0 & 0,00 & 0 & 0,00 & 3 & 4,69 & 3 & 1,73 \\
\hline & A. zenildae & 1 & 2,22 & 1 & 1,92 & 0 & 0,00 & 2 & 3,13 & 4 & 2,31 \\
\hline grandis & A. grandis & 0 & 0,00 & 0 & 0,00 & 0 & 0,00 & 3 & 4,69 & 3 & 1,73 \\
\hline leptozona & A. leptozona & 4 & 8,89 & 0 & 0,00 & 0 & 0,00 & 12 & 18,75 & 16 & 9,26 \\
\hline pseudoparallela & A. dissimilis & 3 & 6,67 & 0 & 0,00 & 0 & 0,00 & 0 & 0,00 & 3 & 1,73 \\
\hline \multirow[t]{3}{*}{ spatulata } & A. manihot & 14 & 31,11 & 33 & 63,46 & 1 & 8,33 & 11 & 17,19 & 59 & 34,10 \\
\hline & A. montei & 2 & 4,44 & 1 & 1,92 & 3 & 25,00 & 3 & 4,69 & 9 & 5,20 \\
\hline & A. pickeli & 6 & 13,33 & 3 & 5,77 & 2 & 16,67 & 13 & 20,31 & 24 & 13,87 \\
\hline Total & & 45 & 100 & 52 & 100 & 12 & 100 & 64 & 100 & 173 & 100 \\
\hline Anastrepha spp. (machos) & & & & & & & & & & 639 & \\
\hline Total geral & & & & & & & & & & 812 & \\
\hline
\end{tabular}


A retirada e a troca do atrativo foram realizadas semanalmente. Os insetos capturados foram retirados da solução das armadilhas através da filtragem e os insetos retidos foram levados ao laboratório para triagem, conservando-se os tefritídeos capturados em vidros contendo álcool $70 \%$ para posterior identificação.

Os exemplares de Anastrepha capturados foram sexados, e os machos identificados até gênero. Para a identificação das espécies, as fêmeas de Anastrepha foram examinadas sob microscópio estereoscópio e submetidas à identificação específica, baseada, principalmente, nas características morfológicas do ápice do acúleo, asas e mediotergito, utilizando-se chaves de identificação (ARAujo et al., 1998; Araujo et al., 1999; ZUCCHI, 2000a).

Foram coletados 812 tefritídeos, sendo 639 machos (78,7\%) e 173 fêmeas (21,3\%) e identificadas 14 espécies de moscas do gênero Anastrepha: A. amita Zucchi, A. dissimilis, A. distincta Greene, A. fraterculus, $A$. grandis, A. leptozona (Hendel), A. manihoti, A. montei Lima, A. obliqua, A. pickeli, A. quiinae, A. sororcula Zucchi, A. turpiniae Stone e A. zenildae, pertencentes a cinco grupos infragenéricos (Tabela 1).

Dentre 19 espécies de Anastrepha registradas no Estado de Goiás (Veloso et al., 2012), aproximadamente $58 \%$ ocorreram neste estudo, sendo assinaladas mais 3 espécies de Anastrepha, aumentando para 22 o número de espécies de moscas-das-frutas detectadas em Goiás.

A espécie alvo do estudo, A. grandis, foi capturada, em baixa prevalência, pela primeira vez nos municípios de Uruana e Jaraguá, nos levantamentos realizados nas safras de 2008 e 2009 nos cultivos de cucurbitáceas em áreas sob SMR. Em Jaraguá foi capturado um macho (0,06 MAD) eem Uruana foram capturadas 3 fêmeas, ocorrendo a primeira captura em novembro de 2008 (0,06 MAD) e as demais em agosto e outubro de 2009 (0,04 e 0,02 MAD, respectivamente). Assim, como a família Cucurbitaceae é hospedeira preferencial dessa espécie, é provávelque o cultivo intensivo desses hospedeiros ao longo dos anos, nas mesmas áreas, venha a favorecer o aparecimento da espécie. Verificou-se que o índice MAD encontrado manteve-se dentro dos índices aceitos na Instrução Normativa $\mathrm{n}^{\mathrm{0}}$ 16, de 5 de março de 2006, o que possibilitou a exportação para Argentina de frutos frescos de melancia, melão e abóbora, oriundos dos municípios de Carmo do Rio Verde, Itapuranga, Jaraguá e Uruana.

Nãofoiconstatadaa presença de mosca-das-frutas nos levantamentos realizados neste estudo, nos anos de 2006 e 2007. Convém ressaltar que, nesses anos, as armadilhas haviam sido instaladas exclusivamente em áreas com plantios de cucurbitáceas. Resultado semelhante foi obtido por Araújo et al. (2009), em que não foi capturada nenhuma espécie de moscas- das-frutas em armadilhas instaladas exclusivamente em cultivos com melão. Porém, MonTes et al. (2011), em cultivos de cucurbitáceas sobSMR em São Paulo, capturaram 48 espécies de Anastrepha, e A. grandis não ocorreu.

Das 14 espécies de Anastrepha identificadas neste trabalho, A. pickeli, A. dissimilis e A. quiinae foram registradas pela primeira vez em Goiás, com uma frequência de $13,87 \%, 1,73 \%$ e 1,73\%,respectivamente (Tabela 1). A ocorrência de $A$. dissimilis foi verificada em diferentes estados brasileiros por vários autores: Trindade; UchôA-Fernandes (2006), URAMOto (2007) e SÁ et al. (2008). A. quiinae foi registrada em Santa Catarina, São Paulo, extremo sul da Bahia e Espírito Santo, por Garcia et al. (2002), SouZa Filho et al. (2003) eURAMOto (2007), respectivamente. Segundo ZucCHI (2007), A pickeli ocorre em 14 estados brasileiros.

Neste estudo, A. manihoti foi a espécie capturada com maior frequência (34,1\%), seguida de A. obliqua $(19,65 \%)$, A. pickeli $(13,87 \%)$ e A. leptozona $(9,26 \%)$ (Tabela 1). A captura destas espécies ocorreu em maior número nos anos de 2004 e 2005, período em que as armadilhas foram instaladas próximas ou em pomares domésticos, contendo frutíferas hospedeiras preferenciais dessas moscas.

A.manihoti, A. obliqua, A. montei e A. pickeli apresentaram ampla distribuição na região do Vale do São Patrício, tendo ocorrido em todos os municípios estudados (Tabela 1). Nesta região, A. obliqua e A. fraterculus foram capturadas nas armadilhas por FELIPE (2004), com baixa frequência nos municípios de Jaraguá e Carmo do Rio Verde. Araújo et al. (2009), em estudos similares a esse trabalho, na região do Baixo Jaguaribe, Ceará, verificaram a ocorrência das espécies $A$. obliqua e $A$. sororcula em todos os municípios amostrados.

As outras espécies capturadas nesta pesquisa que se destacaram foram: A. leptozona $(9,26 \%), A$. fraterculus $(6,95 \%)$, A. montei $(5,20 \%)$, A. zenildae $(2,31 \%)$. As demais espécies apresentaram baixa frequência, com índices inferiores a 2\% (Tabela 1).

A região do Vale do São Patrício apresenta condições para o estabelecimento e manutenção do Sistema de Mitigação de Risco de A. grandis em cultivos de cucurbitáceas. A manutenção deste sistema está condicionada ao monitoramento das moscasdas-frutas a cada safra e cumprimento de demais medidas fitossanitarias, preconizadas no SMR, que conjuntas garantem a segurança fitossanitária dos frutos destinados ao mercado externo.

\section{REFERÊNCIAS}

ARAUJO, E.L.; NASCIMENTO, F.M.; ZUCCHI, R.A. Utilização da análise discriminante em estudos taxonômicos de moscas-das-frutas do gênero Anastrepha 
Schiner, 1868 (Diptera: Tephritidae). Scientia Agricola, v.55, p.105-110, 1998.

ARAUJO, E.L.; VELOSO, V.R.S.; SOUZA FILHO, F.M.; ZUCCHI, R.A. Caracterização taxonômica, novos registros de distribuição e de hospedeiros de Anastrepha turpiniae Stone (Diptera: Tephritidae), no Brasil. Anais da Sociedade Entomológica do Brasil, v.28, n.4, p.657-660, 1999.

ARAUJO, E.L.; CUNHA, A.A.; SILVA, R.K.B.; NUNES, A.M.M.; GUIMARÃES, J.A. Espécies de moscas-dasfrutas (Diptera, Tephritidae) na região do Baixo Jaguaribe, Estado do Ceará. Arquivos do Instituto Biológico, São Paulo, v.76, n.4, p.577-581, 2009. Disponível em: <http://www.biologico.sp.gov.br/docs/arq/v76_4/ araujo2.pdf>. Acesso em: 15 abr. 2013.

BONDAR, G. Moscas de frutas na Bahia. Boletim do Campo, n. 34, p.13-15,1950.

BRASIL. Ministério da Agricultura, Pecuária e Abastecimento. Secretaria de Defesa Agropecuária. Instrução Normativa $\mathrm{n}^{\mathrm{0}} 16$, de 05 de março de 2006. Estabelece, para fins de Certificação Fitossanitária com Declaração Adicional, a condição para Sistema de Mitigação de Risco, como opção reconhecida de manejo de risco para a praga Anastrepha grandis Macquart, em cultivos de Cucumis melo L. (melão), Citrullus lanatus Thunb. (melancia), Cucurbita spp. (abóbora) e Cucumis sativus L. (pepino). Diário Oficial da União, Brasília, DF, 12 de abril de 2006. Seção 1, p.2.

FELIPE, A.F.C.L. Espécies de tripes (Thysanoptera) $e$ moscas-das-frutas Anastrepha grandis (Macquart) (Diptera, Tephritidae) associados à cultura da melancia em Goiás. 2004. 68p. Dissertação (Mestrado em Agronomia: Produção Vegetal) - Escola de Agronomia e Engenharia de Alimentos, Universidade Federal de Goiás, Goiânia, 2004.

GARCIA, F.R.M.; CAMPOS, J.V.; CORSEUIL, E. Lista documentada das moscas-das-frutas (Diptera, Tephritidae) de Santa Catarina, Brasil. Biociências, v.10, n.1, p.139-148, 2002.

GOIÁS. Superintendência de Estatística, Pesquisa e Informações Socioeconômicas. Estatísticas Municipais (Séries Históricas). 2010. Disponível em: <http://www. seplan.go.gov.br/sepin/perfilweb/estatistica_bde.asp>. Acesso em: 5 jul. 2011.

GOIÁS. Agência Goiana de Defesa Agropecuária. Sanidade Vegetal: Programas. Programa de prevenção e controle de pragas em cucurbitáceas. 2013. Disponível em: <http://www.agrodefesa.go.gov.br/>. Acesso em: 15 ago. 2013.

MALAVASI, A. Áreas livres ou de baixa prevalência. In: MALAVASI, A.; ZUCCHI, R.A. (Orgs.). Moscas-das-frutas de importância econômica no Brasil: conhecimento básico e aplicado. Ribeirão Preto: Holos, 2000. cap.23, p.175-181.
MALAVASI, A.; ZUCCHI, R.A.; SUGAYAMA, R.L. Biogeografia. In: MALAVASI, A.; ZUCCHI, R.A. (Org.). Moscas-das-frutas de importância econômica no Brasil: conhecimento básico e aplicado. Ribeirão Preto: Holos, 2000. cap. 10, p.93-98.

MONTES, S.M.N.M.; RAGA, A.; SOUZA-FILHO, M.F. Levantamento de espécies de Anastrepha (Diptera, Tephritidae) em áreas de cucurbitáceas sob Sistema de Mitigação de Risco. Arquivos do Instituto Biológico, São Paulo, v.78, n.2, p.317-320, 2011.

NORRBOM, A.L. The species of Anastrepha (Diptera:Tephritidae) with a grandis-type wing patterns. Proceedings of the Entomological Society of Washington, v.93, p.101-124, 1991.

NORRBOM, A.L.; KORYTKOWSKI, C.A.; ZUCCHI, R.A.; URAMOTO, K.; VENABLE, G.L.; McCORMICK, J.; DALLWITZ, M.J. DALLWITZ. Onwards. Anastrepha and Toxotrypana: descriptions, illustrations, and interactive keys. Version: 31st 2012. Disponivel em: <http:/deltaintkey.com>. Acesso em: 10 abr. 2013.

SÁ, R.F.; CASTELLANI, M.A.; NASCIMENTO, A.S.; BRANDÃO, M.H.S.T.; SILVA, A.N.; PÉREZ-MALUF, R. Índice de infestação e diversidade de moscas-das-frutas em hospedeiros exóticos e nativos no pólo de fruticultura de Anagé, Ba. Bragantia, v.67, n.2, p.401-411, 2008.

SOUZA FILHO, M.F.; RAGA, A.; ZUCCHI, R.A. Moscas-das-frutas no estado de São Paulo: ocorrência e danos. Laranja, v.24, n.1, p.45-69, 2003.

TRINDADE, R.B.R.; UCHÔA-FERNANDES, M.A. Fruit fly species (Diptera: Tephritidae) in the Amazonian Forest at Oiapoque region, Amapá state, Brazil. In: INTERNATIONAL SYMPOSIUM ON FRUIT FLIES OF ECONOMIC IMPORTANCE, 7., 2006, Salvador, BA. Proceedings. Salvador: 2006. 1 CD-ROM.

URAMOTO, K. Diversidade de moscas-das-frutas (Diptera, Tephritidae) em pomares comerciais de papaia e em áreas remanescentes da Mata Atlântica e suas plantas hospedeiras nativas, no município de Linhares, Espírito Santos. 2007. 105p. Tese (Doutorado em Ciências: Entomologia) Escola de Superior de Agricultura Luiz de Queiroz, Universidade de São Paulo, Piracicaba, 2007.

VELOSO, V.R.S.; FERNANDES, P.M.; ZUCCHI, R.A. Moscas-das-frutas nos estados brasileiros: Goiás. In: MALAVASI, A.; ZUCCHI, R.A. (Orgs.). Moscas-das-frutas de importância econômica no Brasil: conhecimento básico e aplicado. Ribeirão Preto: Holos, 2000. cap. 23, p.175-181.

VELOSO, V.R.S.; PEREIRA, A.F.; FERREIRA, G.A; BRAGA FILHO, J.R.; FELIPE, A.F.C.L.; FERREIRA, H.J. Ocorrência e distribuição de Anastrepha grandis (Macquart) (Diptera, Tephritidae) no Estado de Goiás e Tocantins, Brasil. In: JORNADA CIENTÍFICA DA PÓS-GRADUAÇÃO NA ESCOLA DE AGRONOMIA E 
ENGENHARIA DE ALIMENTOS/UFG, 2., 2005, Goiânia. Resumos. UFG: 2005. 1 CD-ROM.

VELOSO, V.R.S.; PEREIRA, A.F.; RABELO, L.R.S.; CAIXETA, C.V.D.; FERREIRA, G.A. Moscas-das-frutas (Diptera, Tephritidae) no Estado de Goiás: ocorrência e distribuição. Pesquisa Agropecuária Tropical, v. 42, n.3, p.357-367, 2012.

ZUCCHI, R.A. Moscas-das-frutas (Diptera, Tephritidae) no Brasil: taxonomia, distribuição geográfica e hospedeiros. In: ENCONTRO SOBRE MOSCAS-DAS-FRUTAS, 1., 1987, Campinas. Anais. Campinas: 1988. p.1-10.

ZUCCHI, R.A. Taxonomia. In: MALAVASI, A.; ZUCCHI, R.A. (Orgs.). Moscas-das-frutas de importância econômica no Brasil: conhecimento básico e aplicado. Ribeirão Preto: Holos, 2000a. cap. 1, p.13-24.

ZUCCHI, R.A. Espécies de Anastrepha, sinonímias, plantas hospedeiras e parasitóides. In: MALAVASI, A.; ZUCCHI, R.A. (Orgs.). Moscas-das-frutas de importância econômica no Brasil: conhecimento básico e aplicado. Ribeirão Preto: Holos, 2000b. cap. 4, p.412-418.

ZUCCHI, R.A. Mosca-do-mediterrâneo, Ceratitis capitata (Diptera, Tephritidae). In: VILELA, E.F.; ZUCCHI, R.A.; CANTOR, F. (Orgs.). Histórico e impacto das pragas introduzidas no Brasil. Ribeirão Preto: Holos, 2001. p.15-22.

ZUCCHI, R.A. Diversidad, distribución y hospederos del género Anastrepha en Brasil. In: ORTIZ-HERNANDÉZ, V. Moscas de la fruta en Latinoamérica (Diptera, Tephritidae): diversidad, biología y manejo. México, DF: S y G Editores, 2007. p.77-100.

ZUCCHI, R.A. Fruit flies in Brazil. Anastrepha species their host plants and parasitoids. 2008. Disponível em: <http://www.lea.esalq.usp.br/anastrepha/>. Atualizado em 12 de abril de 2013. Acesso em: 14 abr. 2013.

Recebido em 13/9/11

Aceito em 16/4/12 